\title{
Niboshi for Slate Devices: A Japanese Input Method Using Multi-touch for Slate Devices
}

\author{
Gimpei Kimioka, Buntarou Shizuki, and Jiro Tanaka \\ Department of Computer Science, University of Tsukuba, \\ SB1024 University of Tsukuba, 1-1-1 Tennodai, Tsukuba-shi, Ibaraki, Japan \\ \{gimpei, shizuki, jiro\}@iplab.cs.tsukuba.ac.jp
}

\begin{abstract}
We present Niboshi for slate devices, an input system that utilizes a multi-touch interface. Users hold the device with both hands and use both thumbs to input a character in this system. Niboshi for slate devices has four features that improve the performance of inputting text to slate devices: it has a multi-touch input, enables the device to be firmly held with both hands while text is input, can be used without visual confirmation of the input buttons, and has a large text display area with a small interface. The Niboshi system will enable users to type faster and requires less user attention to typing than existing methods.
\end{abstract}

\section{Introduction}

Slate devices, such as the iPad or the Galaxy Tab, are mobile devices that have an approximately 7 by 10 inch touchscreen as the main interface. They have more possible applications and bigger screens than smartphones or PDAs, so they are becoming increasingly widely used. Users tend to input text more on these devices than they do on smartphones or PDAs.

Onscreen QWERTY keyboard (QWERTY) is an existing method commonly used to input Japanese on slate devices. Users input Japanese text by using the alphabet to spell out Hiragana characters phonetically, just like with a hardware-based QWERTY keyboard. For example, 日本 is a kanji word that means Japan, and pronounced as Nippon. This word can be input the pronunciation with Alphabet and converted into kanji afterwards. We believe that this technique is inefficient since it simply mimics a hardware-based QWERTY keyboard and does not consider the characteristics of slate devices. There are three problems with using QWERTY on slate devices.

- Users cannot input text while holding the device firmly. QWERTY is efficient only if the device is put onto a desk or table since all the fingers of both hands are required in order to use it as if it were a hardware-based QWERTY keyboard. There are many situations in which users may not have access to a table when using the device, such as when standing in a train or sitting on a bench. In those cases, users have to hold the device with both hands, or hold it with one hand and use only the other hand to input text. Slate devices are heavier than smartphones or PDAs, which means their stability while they are held is compromised, or only one hand can be used for inputting. 
- Users need to look at both the text display area and the input buttons while they are inputting. Since QWERTY does not offer tactile feedback to users when buttons are touched, users have to look at the button they want to push.

- The user interface dominates part of the screen because it has to be big enough to be consistent with the hardware-based QWERTY keyboard. This decreases the visual accessibility of the document when the document is long and cannot be displayed without scrolling.

Since QWERTY is not quite optimized for slate devices as these show, we solved those problems by developing a new Japanese input system specialized for slate devices.

We have been developing a Japanese input system that utilizes a multi-touch interface for smartphones [1]. This system enables users to input a series of Japanese characters with both thumbs simultaneously. In this paper, we present Niboshi for slate devices, which is a Japanese input system modified from our previous work on smartphones.

Niboshi for slate devices uses a multi-touch interface, as our previous system does. The multi-touch interface enables users to input a character as quickly as by one stroke of one finger, once they have been trained in its use. The system also enables characters to be input without the user having to visually confirm the button being pressed because its button layout can be calibrated and the system also provides visual feedback.
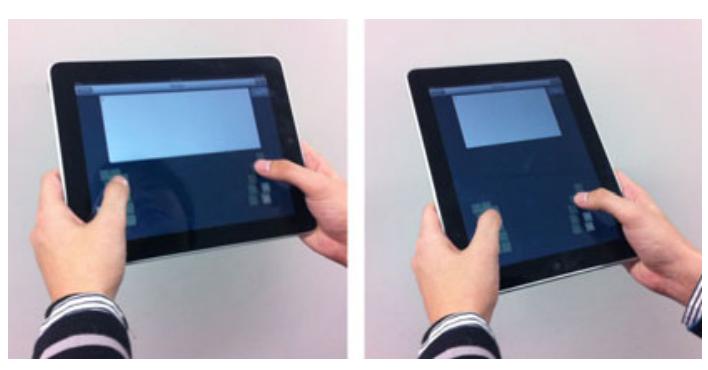

Fig. 1. How to hold device in portrait mode (left) and landscape mode (right)

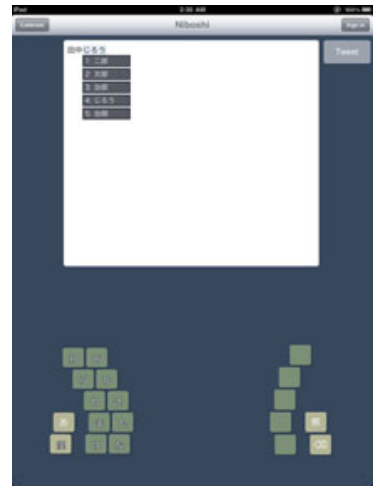

Fig. 2. Interface of Niboshi

\section{Related Works}

There are related works about text input systems on touch screen devices, kanji conversion system and multi-touch input system. Modified button layouts on touch screen devices are presented in OPTI [2] and Metropolis [3]. Shiohara et al. presented a button layout for Japanese input system that considers kinematic characteristics of thumb movements [4]. This system is about single handed input and our system uses the button layout that can be calibrated for both hands. TagType [5] suggests that 
users use both thumbs to input Japanese text. TagType can only handle one input at a time, and our system uses multi-touch. Shin et al. presented a multi-touch Korean text input system that reduces the stroke number of input [6]. This system is for Korean and uses the multi-touch to add the variety of consonants.

\section{Niboshi for Slate Devices}

Our Japanese input system, Niboshi for slate devices, is described in more detail in this section.

\subsection{How to Hold Device}

In Niboshi for slate devices (Niboshi), the device is held with both hands. Users can hold the device in portrait or landscape mode (Fig. 1). In portrait mode, more text is visually accessible because the screen can show more lines. In landscape mode, the device can be held more stably because the device's center of gravity is between the hands. Users can simply hold the device as they want, and the system will recognize the orientation and provide the appropriate interface.

\subsection{User Interface}

The interface of Niboshi is shown in Fig. 2. The buttons are divided into two sets, and each set to appear directly under both thumbs. Consonant buttons are on the left, and vowel buttons are on the right. Function buttons, such as delete button, are placed in relation to the other buttons.

Since users have different sized hands and may hold different parts of the device, the location of the buttons can be calibrated specifically for the user. Niboshi has the calibration function that is explained later in this paper.

\subsection{How to Input}

The written Japanese language basically consists of three types of character sets: hiragana, katakana, and kanji. Hiragana and katakana are phonetic characters and are phonetically interchangeable. These characters generally start with a consonant and end with a vowel sound. In Niboshi, users can input hiragana and katakana in the same way. They may choose hiragana or katakana before they start inputting. Kanji can be converted from hiragana/katakana depending on how they are pronounced.

Both hiragana and katakana have several types of sounds: voiceless sounds, voiced consonant sounds, p-consonant sounds, contracted sounds, and a double consonant. In Niboshi, voiceless sounds are input in a basic way, and the other sounds can be converted from the voiceless sounds as the diverted sound from voiceless sound.

Voiceless Sounds. For a voiceless sound, a user inputs a hiragana/katakana by choosing a consonant with his/her left thumb and a vowel with his/her right thumb. Character input is confirmed only by the right thumb, so users do not have to cancel an input even if the wrong consonant was chosen. Both hiragana and katakana can be input using the procedure shown below and in Fig. 3. 
1. Choose a consonant with your left thumb and press on it. The right-side buttons vary depending on which consonant is being pressed (Fig. 3a).

2. Choose a vowel with your right thumb. The text display field shows the hiragana or katakana corresponding to the combination of the consonant and the vowel you are pressing, so you do not have to visually confirm which buttons you are holding (Fig. 3b).

3. Release your right thumb to confirm the character. The left thumb does not have to be released, which reduces the actions required to continue inputting text (Fig. 3c).

4. Move your left thumb to the consonant button that you want to input next. Repeat the procedure from step 1 (Fig. 3d).

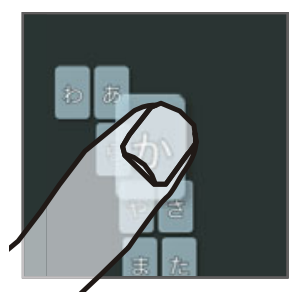

(a) Choose a consonant with left thumb and hold.

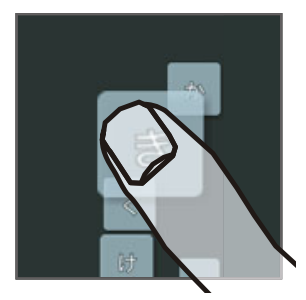

(b) Choose a vowel with right thumb.

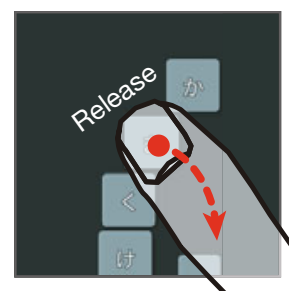

(c) Release right thumb and confirm the character.

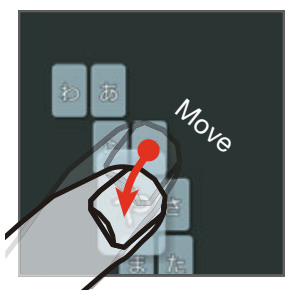

(d) Choose the next consonant and go back to (a).

Fig. 3. How to input hiragana/katakana

Table 1 shows what character will be input from which combination of consonant and vowel. All voiceless consonant sounds in Japanese are determined from these combinations.

Table 1. Voiceless sound combinations

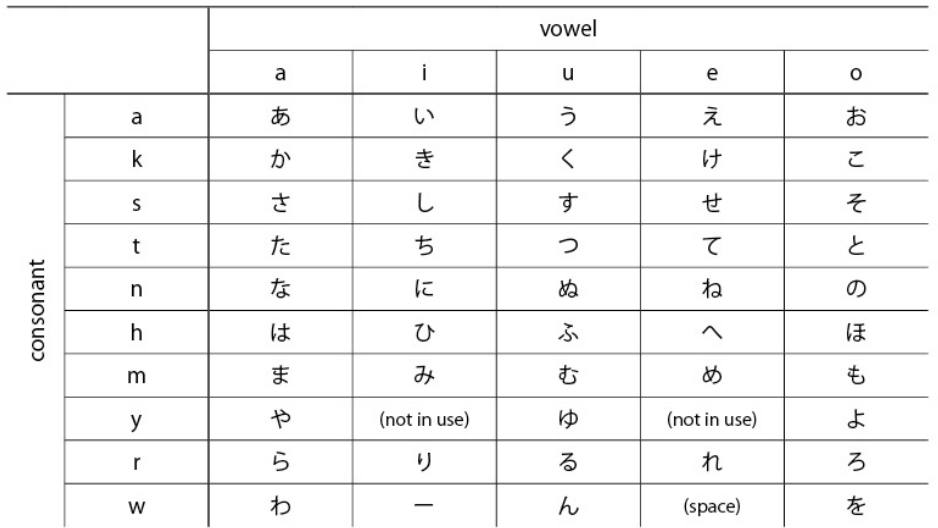

Other Sounds. Sounds other than voiceless sounds are phonetically diverted from voiceless sounds. A gesture is introduced to convert a voiceless sound into a diverted sound. This reduces the number of strokes needed to input a character. The gesture 
continuously follows the right thumb touch so that the user can confirm a character and choose the diverted sounds at the same time.

The gesture to convert a sound is performed by the right thumb. The user slides the thumb in the direction of the thumb joint (Fig. 4). Users may also use the MicroRolls [7] gesture (Fig. 4). MicroRolls is a way for users to roll their thumbs up/down on the screen.

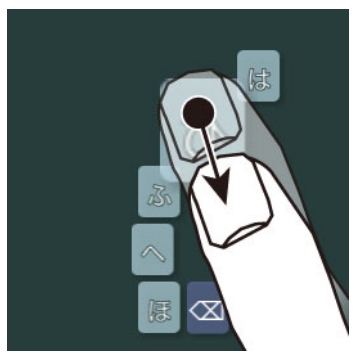

Fig. 4. Gesture for converting sound
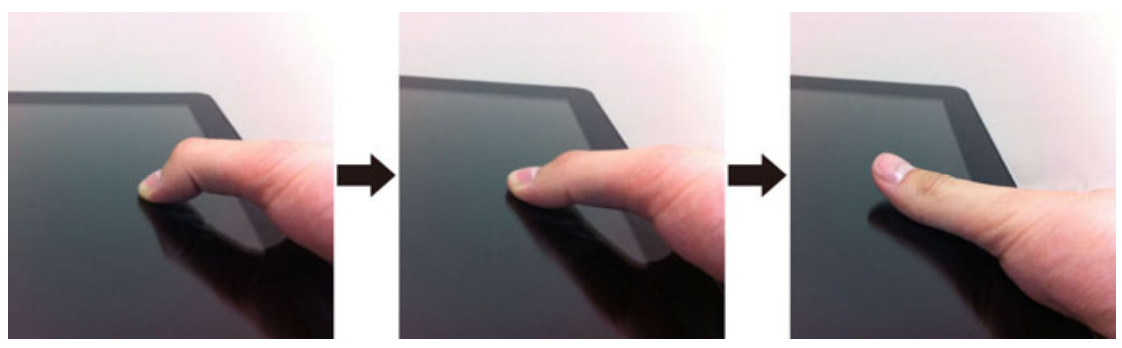

Fig. 5. MicroRolls gesture

Kanji Conversion. One of the Japanese language characteristics is that it has a lot of homonyms, and one kanji word cannot be determined phonetically. Many Japanese input systems adopt the way that the system shows several candidates of the kanji word and let the user choose to determine what kanji to input. We also use this technique in Niboshi.

In POBox [8], the system dynamically presents kanji conversion candidates every time a user input a hiragana. In our system, the conversion candidates are shown every time a user input a character like POBox. The system predicts the converted kanji word from what the user has input and show them as the candidates.

Five candidates are shown at a time close to the text input area. The candidates are always shown when the user input, and user can confirm the input whenever he/she wants. To confirm the conversion, the user can hold the conversion button next to consonant buttons, and press the vowel button that corresponds to the candidate he/she wants to choose.

This conversion could be done several times for one input. For example, the 'ha' sound has two diversions: 'ba' and 'pa'. A user simply does the gesture back and forth before releasing his/her thumb, and the system switches the diversion every time it recognizes the gesture. 


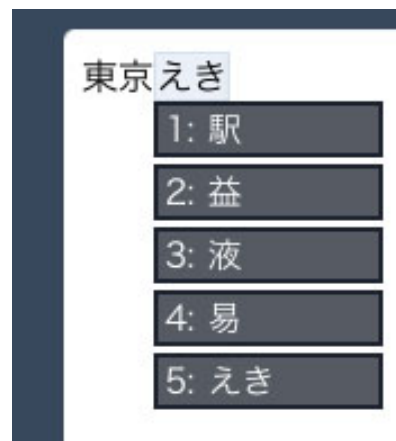

Fig. 6. Kanji conversion candidates

\subsection{Features of Niboshi}

Niboshi has four main features that enable users to input Japanese efficiently on slate devices. We prioritized experienced users as the target users of the system, so the features are aimed towards them rather than novice users. However, since the system is designed to be simple and easy to learn, novice users can quickly learn how to use it.

The four main features of Niboshi are that is has multi-touch input, enables the device to be firmly held with both hands while text is input, can be used without visual confirmation of the input buttons, and has a large text display area with a small interface.

\section{- Multi-touch input}

Multi-touch input in Niboshi enables experienced users to input a character as quickly as by one stroke of one finger. The order in which the consonant and vowel are touched does not matter because the system has robustness for the touch order.

\section{- Device can be firmly held with both hands}

Niboshi is designed so that users can still hold the device with both hands while using Niboshi. All of the input operations can be done by two thumbs, without change to how the device is held.

\section{- Visual confirmation of buttons unnecessary}

Niboshi has a calibration function for aligning the input buttons to the locus of where the user's thumbs touch. The system also uses visual feedback in the text display field to show which buttons the user is holding, so characters can be input without visual confirmation of the buttons being pressed. Both the calibrated button layout and the visual feedback in the text display field have the effect to reduce the number of Focus Of Attention (FOA) [9]. QWERTY requires users to view both the text display field and the software keyboard. In contrast, Niboshi requires users to only view the text display field; this can reduce the stress and improve the speed of inputting text.

\section{- Large text input display area}

The buttons in Niboshi are separated into two sets, one at either edge of the screen. This leaves the middle of the screen open for the text display area, unlike QWERTY, which takes up a full rectangle of space on the screen. 


\subsection{Calibration}

There are more ways of holding slate devices than smartphones or PDAs because slate devices are bigger, thus offering more places for the hands to grasp. They are also heavier, so how they are held depends on the size and the shape of the user's hands.

Therefore, we found that the button layout should be able to be calibrated for each user. We implemented a calibration function in Niboshi by which the system can adjust its button layout for each user depending on the kinematic characteristics of human thumbs. This enables users to hold the device as they want, and thus the system is flexible as to how and where it is held.

To calibrate the system, users hold the device in both hands as they want and are then directed to move their thumbs on the screen (Fig. 7a). Then, the system calculates a button layout from the paths of the thumbs (Fig. 7b).
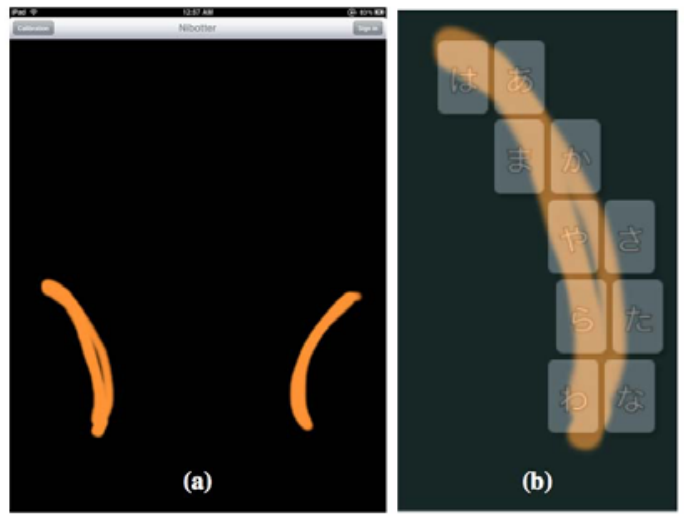

Fig. 7. User's thumb path on screen (a) and calibrated button layout (b)

\section{Implementation}

We have implemented Niboshi in Objective-C and iOS SDK 4.2. The application runs as Twitter client on iPad. The application consists of the interface, hiragana/katakana input engine, kanji conversion, button calibration and twitter engine. Sticky hit check function is implemented to stabilize the hit check from two hands. We found that a user often input a character that he/she didn't intend in a pilot study. This is because a point touched by a thumb moves small amount when the other thumb releasing. To avoid this problem, we implemented sticky hit check. Sticky hit check is the function that enlarges the hit check area of a button when the button is touched (Fig. 8). 


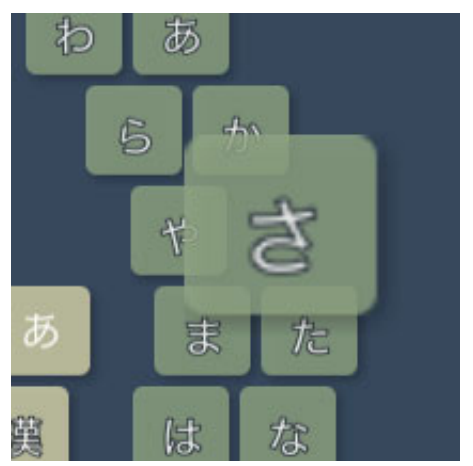

Fig. 8. Sticky hit check

\section{Evaluation}

We conducted an experiment with one user to evaluate the learning curve of Niboshi. The subject is one of the authors who is used to computer operation. The task of the experiment is text-copying. The time to copy an article (206 characters long) from a news web site using Niboshi, and he is instructed to do at least one session every day,. The experiment was conducted for 56 sessions for 56 days.

\subsection{Results of the Experiment}

Fig. 9 shows the result of the experiment, and indicates the speed of input using Niboshi. The horizontal axis shows the sessions, and the vertical axis shows the number of characters input per minute (cpm).

We estimate the learning curve of this experiment from power trendline of the result that is shown as bold line in Fig. 9. The learning curve shows that our system can be fairly learnt in continuous usage.

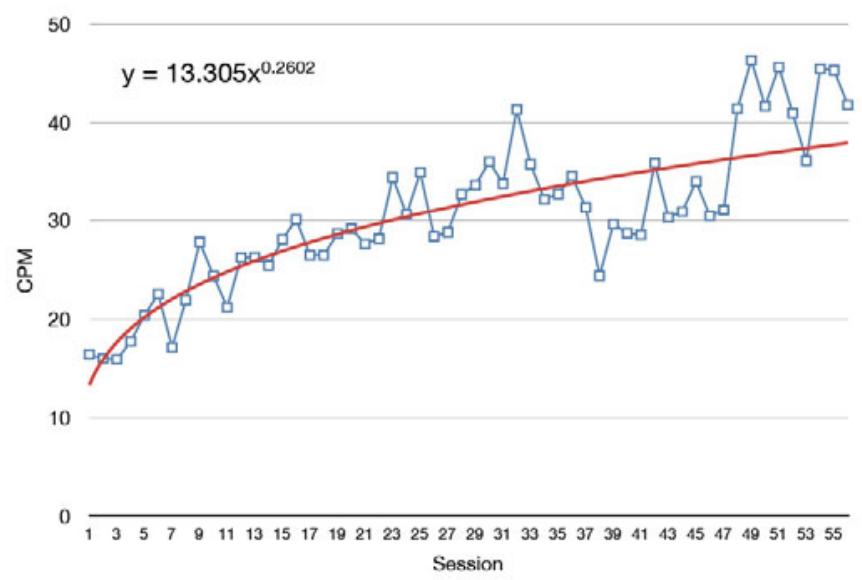

Fig. 9. Input speed of Niboshi 
The maximum input speed through the experiment was $46.3 \mathrm{cpm}$. The input speed increases as the session goes, so we believe that users can learn how to input in Niboshi quickly enough.

\section{Conclusion}

We developed Niboshi, a Japanese input method for slate devices. Niboshi has four features that improve the overall performance of Japanese input on slate devices. We also conducted an experiment and made sure the learning rate of the system and it indicates that the system can be learnt fast enough to be used on daily basis.

\section{References}

1. Kimioka, G., Shizuki, B., Tanaka, J.: Japanese input method using multi-touch for mobile devices and its evaluation. In: IPSJ SIG Technical Report vol. 2010-HCI-138 pp.1-6 (2010)

2. Blickenstorfer, C.H.: Graffiti: In Wow! Pen Computing Magazine, pp. 30-31 (1995)

3. Zhai, S., Hunter, M., Smith, B.A.: The metropolis keyboard - an exploration of quantitative techniques for virtual keyboard design. In: Proceedings of the 13th Annual ACM Symposium on User Interface Software and Technology, UIST 2000, New York, pp. 119-128 (2000)

4. Shiohara, Y., Tano, S., Ichino, J., Hashiyama, T.: Eyes-free one stroke thumb text entry based on the movement characteristic. In: Proceedings of Human Interface Symposium 2007, pp. 573-576 (2007)

5. Tanaka, M., Tagawa, K., Yamanaka, S.: A study on developing of new thumb keyboard TagType. In: Proceedings of the Japan Society of Mechanical Engineers 2001, pp. 112-116 (2001)

6. Shin, H., Lee, W., Lee, G., Cho, I.: Multi-point touch input method for korean text entry. In: Proceedings of the 27th International Conference Extended Abstracts on Human Factors in Computing Systems, CHI 2009, New York, pp. 3871-3876 (2009)

7. Roudaut, A., Lecolinet, E., Guiard, Y.: Microrolls: expanding touch-screen input vocabulary by distinguishing rolls vs. slides of the thumb. In: Proceedings of the 27th International Conference on Human Factors in Computing Systems, CHI 2009, New York, pp. 927-936 (2009)

8. Masui, T.: A Fast Text Input Method for Pen-based Computers. In: Proceedings of Workshop on Interactive Systems and Software 1997, pp. 51-60 (1997)

9. Mackenzie, I.S., Tanaka-Ishii, K.: Text Entry Systems. Morgan Kaufmann, San Francisco (2007) 